\title{
Preliminary data on the presence of bacteria in the uterus of pregnant cows
}

Pedersen, Hanne Gervi; Knudsen, L.R.V. ; Agerholm, Jørgen Steen; Jensen, T.K.; Klitgaard, K.S.; Karstrup, Cecilia Christensen

Publication date:

2015

Citation for published version (APA):

Pedersen, H. G., Knudsen, L. R. V., Agerholm, J. S., Jensen, T. K., Klitgaard, K. S., \& Karstrup, C. C. (2015).

Preliminary data on the presence of bacteria in the uterus of pregnant cows. Abstract from IETS, Louisville,

Kentucky, United States. 
indicating that other genital system organs were not formed. For the 30 days of development of guinea pigs, we observed that gonadal cords were differentiated in testicular cords by invasion of mesenchymal and endothelial cells, and also composed of Sertoli cells and primordial germ cells. These cords were among a large amount of testicular mesenchyme at the 40-day group. With 50- and 65-day development samples, the gonad was completely differentiated into testicle, with the presence of spermatogonia and Sertoli cells in the seminiferous tubules, and a large amoun of interstitial Leydig cells around the tubules. We conclude that gonadal differentiation in guinea pig males occurs around the middle of pregnancy, between 25 and 30 days and that, before the end of the pregnancy, at 50 days, the testicle presents morphology similar to that found in the
postnatal period.

\title{
Early Pregnancy
}

\section{CIRCULATING micrORNAS AS POTENTIAL BIOMARKERS OF EARLY PREGNANCY IN HIGH-PRODUCING DAIRY COWS}

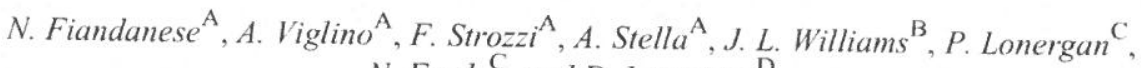 \\ N. Forde ${ }^{\mathrm{C}}$, and D. Iamartino ${ }^{\mathrm{D}}$

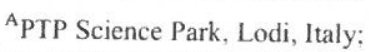 \\ ${ }^{\mathrm{B}}$ School of Animal and Veterinary Sciences, University of Adelaide. Roseworthy, Australia; \\ ${ }^{\mathrm{C}}$ School of Agriculture and Food Science, University College Dublin, Dublin, Ireland; \\ ${ }^{\mathrm{D}}$ Associazione Italiana Allevatori, Laboratorio Genetica e Servizi, Cremona, Italy
}

\begin{abstract}
Lactation induces changes in the metabolic status of postpartum dairy cows that negatively affects the likelihood of pregnancy establishment. At present, pregnancy diagnosis with confidence is only possible after the third week of gestation. Earlier diagnosis could facilitate earlier re-breeding, reduce calving intervals, and improve profits for the industry. MicroRNAs (miRNAs) released in body fluids have been identified as minimally invasive biomarkers of several diseases. In addition, distinct miRNA profiles have been directly related to specific stages of human pregnancy. The aim of this study was to profile circulating miRNAs in the blood of high-producing dairy cows in order to identify biomarkers of early pregnancy. In-calf primiparous Holstein-Friesian cows $(n=22)$ with a similar economic breeding index were used. At calving, cows were randomly assigned to one of two groups: (1) lactating $(n=11$; milked twice per day) or (2) non-lactating $(n=11$; dried off immediately). Around 65 to 75 days postpartum (dpp), oestrous cycles were synchronized and a single embryo from a superovulated Holstein-Friesian donor was transferred at Day 7 post-oestrus. Plasma samples were analysed at Day 13 (initiation of conceptus elongation) and at Day 19 (initiation of implantation). Pregnancy rate, established by the presence of conceptus at Day 19, was 5/11 (45\%) for lactating and $8 / 11(73 \%)$ for non-lactating cows, respectively. Circulating miRNA levels were profiled in 4 animals per group in non-lactating pregnant, and lactating pregnant and nonpregnant cows at selected timepoints using Illumina HiSEqn 2000 (Illumina Inc.. San Diego, CA, USA) for smallRNA sequencing. Annotation and discovery of miRNAs were done using MirDeep2, and read counts were analysed using edgeR to identify differentially expressed miRNAs. Target genes analysis was run with miRWalk and pathways interactions were built using Cytoscape $(P \leq 0.05)$. Differentially abundant miRNAs between lactating and non-lactating cows were found at both time points (FDR $\leq 0.05$ ). At Day 13. non-lactating cows had a distinct miRNA profile compared with lactating cows showing 8 differentially expressed miRNA ( $6 v$. pregnant and $2 v$ nonpregnant cows). At Day 19. no significant differences were found within pregnant cows, but 5 differentially expressed miRNAs were identified between pregnant and nonpregnant cows, regardless of metabolic status. Interestingly, one miRNA, bta-mir140, was up-regulated in non-lactating pregnant cows from Day 13 onwards compared with nonpregnant cows. Furthermore, the same miRNA was up-regulated in lactating pregnant $v$. nonpregnant at Day 19. Among bta-mir 140 target genes, CD274, SLC 44A4, CXCL12, and SIRPA were strictly associated with immune tolerance. In conclusion, the maternal plasma miRNome may represent an early indicator of pregnancy status. In particular, the up-regulation of bta-mir 140 in pregnant cows suggests that this miRNA may be a good candidate as an early biomarker of fertility. Furthermore, the positive correlation between this miRNA and pathways involved in T-cell response may indicate a role of immune tolerance in preventing rejection at the initiation of implantation.
\end{abstract}

\section{PRELIMINARY DATA ON THE PRESENCE OF BACTERIA IN THE UTERUS} OF PREGNANT COWS

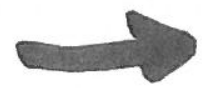

H. G. Pedersen ${ }^{\text {A }}$, L. R. V. Knudsen ${ }^{\mathrm{B}}$, J.S. Agerholm ${ }^{\mathrm{A}}$, T. K. Jensen ${ }^{\mathrm{B}}$, K. S. Klitgaurd ${ }^{\mathrm{B}}$, and C. C. Karstrup ${ }^{\mathrm{A}}$

${ }^{A}$ Department of Large Animal Sciences, Faculty of Health and Medical Sciences, University of Copenhagen, Frederiksberg, Denmark:

${ }^{\mathrm{B}}$ National Veterinary Institute. Technical University of Denmark. Frederiksberg, Denmark

Bacterial invasion of the uterus during the postpartum period has been well described. Recent papers using $16 \mathrm{~S} r \mathrm{RNA}$ gene sequencing technique suggest that the nonpregnant uterus contains a diverse flora of bacteria that are not necessarily pathogenic. In contrast. the pregnant uterus has until now been considered a sterile environment. The aim of the present study was to investigate whether bacteria were present in the uteri of pregnan cows. Uteri from pregnant, slaughtered animals $(n=47)$ were sampled. The surface of the uterus was wiped with alcohol, flame sterilized, and cut open with sterile scissors. Samples were taken from the endometrium and from the placentomes. The samples were embedded in paraffin, sectioned at 3 microns, and prepared for fluorescence in situ hybridization using a probe targeting the $16 \mathrm{~S}$ rRNA of the domain bacteria. so that all bacteria regardless of species were visualised. Using fluorescence microscopy, the presence of bacteria within or on the surface of the endometrium and within the placentomes was noted. The stage of pregnancy was estimated to range from 26 to 263 days by measuring the size of the embryo or fetus. The 
endometrial samples from $85.1 \%$ (40/47) of pregnant cows contained bacteria. In 22 cows, the bacteria were localised within the endometrial tissue, whereas in the remaining 18 cows, the bacteria were on the epithelial surface. Placental samples were obtained from 43 cows, and $76.7 \%(33 / 43)$ of these contained bacteria. The presence of bacteria in the pregnant uterus may suggest that a cow can carry a pregnancy despite the presence of few potentially pathogenic bacteria or that normal flora exist in the uterus as in, for example, the vagina. In conclusion, bacteria were present in the endometrium and placentomes of pregnant cows. Further analyses using rRNA gene sequencing techniques will aim to confirm the presence of bacteria in the bovine pregnant uterus and to investigate which species of bacteria are present in the uterus during pregnancy.

73 PROTEOMIC ANALYSIS OF UTERINE LUMINAL FLUID ON DAY 7 OF PREGNANCY IN CATTLE

\author{
C. Passaro ${ }^{\mathrm{A}}$, N. Forde ${ }^{\mathrm{B}}$, T. E. Spencer ${ }^{\mathrm{C}}$, and P. Lonergan ${ }^{\mathrm{A}}$

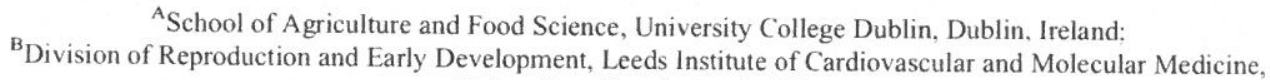 \\ University of Leeds. Leeds, UK; \\ ${ }^{\mathrm{C}}$ Division of Animal Sciences, University of Missouri, Columbia, MO, USA
}

In vitro fertilization studies have demonstrated that contact with the female reproductive tract is not necessary in order for the embryo to reach the hatched blastocyst stage. Furthermore, the endometrial transcriptome does not differ between cyclic and pregnant cattle before maternal recognition around Days 15 to 16 , suggesting that before that time the cow does not know she is pregnant. Nonetheless, embryo quality is significantly improved by exposure to reproductive tract secretions. Therefore, the aims of this study were to (1) characterise the protein content of uterine luminal fluid (ULF) on Day 7 of pregnancy and the oestrous cycle; (2) identify if differences in ULF composition exist between pregnant and cyclic heifers on Day 7; and (3) determine if blastocyst-derived proteins contribute to ULF composition. Following oestrus synchronization, heifers observed in standing oestrus were randomly assigned to either inseminated (AI: $n=12$ ) or cyclic control (C: $n=6)$ groups. At slaughter, the uterine horn ipsilateral to the corpus luteum was flushed with $10 \mathrm{~mL}$ of PBS. In the Al group, the presence of an appropriately developed embryo was noted $(n=8)$, and the ULF of both confirmed pregnant and cyclic heifers was clarified by centrifugation at $1000 \times g$ for 15 min at $4{ }^{\circ} \mathrm{C}$. Blastocyst-conditioned medium was produced by culturing in vitro-produced blastocysts $(n=300)$ for $24 \mathrm{~h}$ in synthetic oviduct fluid (SOF; groups of 50 blastocysts in $500 \mu \mathrm{L}$ of SOF). After $24 \mathrm{~h}$ the conditioned medium $(n=6$ pools), along with contemporaneous blanks ( $n=6)$, was collected and snap frozen. Proteomic analysis of ULF as well as blastocyst-conditioned medium was carried out by nano-LC tandem mass spectrometry (nano-LC-MS/MS). A total of 661 proteins were identified in the ULF of heifers on Day 7 with $>95 \%$ confidence in at least 4 out of the 6 animals analysed per group. The most abundant proteins were myosin-9, cytoplasmic dynein 1 heavy chain 1-like, fatty acid synthase isoform X1, serum albumin, and aminopeptidase $\mathrm{N}$. No differences were detected between pregnant and cyclic ULF. Of the proteins identified, the biological processes of gene expression (23.8\%), small molecule metabolic process $(22.6 \%)$, and cellular protein metabolic process $(17.8 \%)$ were most overrepresented. Interestingly, 400 proteins were associated with extracellular exosome as well as extracellular space ( 87 proteins) and extracellular region (58). Analysis of blastocystconditioned medium identified 11 proteins that were not detected in the contemporaneous blanks, of which one ( $\alpha$-1-acid glycoprotein) was also detected in Day 7 ULF. In conclusion, no differences in the ULF composition between pregnant and cyclic heifers were observed on Day 7 , consistent with studies on the endometrial transcriptome. A large proportion of the proteins detected are associated with the extracellular space region and may represent candidate proteins that can be added to embryo culture media to enhance early development.

Research was funded by Science Foundation Ireland (13/1A/1983).

\title{
74 ANALYSIS OF STEROID HORMONES IN BOVINE OVIDUCTAL FLUID BY GAS CHROMATOGRAPHY COUPLED WITH TANDEM MASS SPECTROMETRY
}

\author{
J. Lamy ${ }^{\mathrm{A}}$, P. Liere $^{\mathrm{B}}$, P. Mermillod ${ }^{\mathrm{A}}$, and M. Saint-Dizier A.C \\ Anstitut National de la Recherche Agronomique, Nouzilly, France; \\ ${ }^{\mathrm{B}}$ Institut National de la Santé et de la Recherche Médicale, Le Kremlin-Bicêtre, France; \\ ${ }^{\mathrm{C}}$ Université François Rabelais, Tours, France
}

Steroid hormones play key roles in the regulation of physiological changes in the mammalian genital tract, including the ${ }^{6}$ viduct. The aim of this study was to determine the variations in steroid hormone concentrations in bovine oviducal fluid (OF) during the oestrous cycle. Bovine oviducts were collected at a local slaughterhouse and classified into 4 stages according to the ovarian and corpus luteum $(\mathrm{CL}) \mathrm{morphologies}(n=18-25 \mathrm{cow} / \mathrm{stage})$ post-ovulatory (postov), mid luteal (mid-lut). late luteal (late-lut). and preovulatory (preov). Follicular fluid was also collected from the preov follicles. Animals with follicular oestradiol- $17 \beta(\mathrm{E} 2)<40 \mathrm{ng} \mathrm{mL} \mathrm{m}^{-1}$ or progesterone (P4) $>160 \mathrm{ng} \mathrm{mL}^{-1}$ (cystic follicles) or E2: $\mathrm{P} 4$ ratio $<1$ (atretic follicles) were excluded. Oviducal fluids were collected from contra- and ipsilateral (to the $\mathrm{CL}$ ) ampullas by squeezing. They were then centrifuged and stored at $-80^{\circ} \mathrm{C}$. Steroids were extracted from pools of 150 to $200 \mu \mathrm{L}$ of OF (4-10 cows/pool; $3-4$ pools per "stage $\times$ side" group), purified. fractioned by high-performance liquid chromatography, derivatized, and analysed by gas chromatography coupled with tandem mass spectrometry (GC-MS/MS). Comparisons between groups were made with a two-way ANOVA followed by Bonferroni post-tests. Data are expressed as means 1 SEM. A $P$-value $<0.05$ was considered as significant. In ipsilateral $\mathrm{OF}$, the concentrations of $\mathrm{P} 4$ increased from postov (56.9 \pm 13.4 ng mL 1 ) to

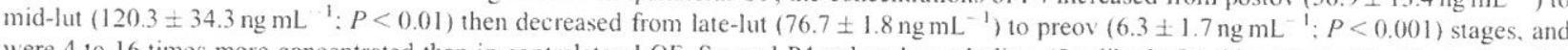

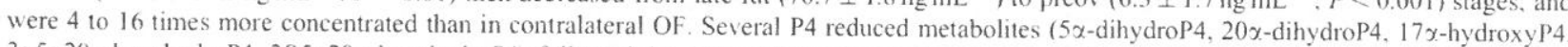
$3 \times 5 \times 20 x$-hexahydroP4, $3 \beta 5 \times 20 x$-hexahydroP4) followed the same pattern of variation. Concentrations of the P4 precursor pregnenolone were highest at postov $(4.2 \pm 0.3 \mathrm{ng} \mathrm{mL}-1$ in ipsilateral $\mathrm{OF})$ then decreased from mid-lut to preov $\left(3.4 \pm 0.6\right.$ to $\left.1.3 \pm 0.1 \mathrm{ng} \mathrm{mL}^{-1} ; P<0.0() \mathrm{l}\right)$ and were 